\title{
Aneurismas de aorta abdominal pequeños: no hubo diferencias entre la cirugía y la conducta expectante
}

Inmediate repair compared with surveillance of small abdominal aortic aneurysms. Lederle $F$, Wilson $S$, Johnson $G$, et al.for the Aneurysm Detection and Management Affairs Cooperative Study Group (ADAM). N Engl J Med 2002;346:1437-44

\section{Objetivo}

Evaluar si la cirugía electiva temprana de las aneurismas de aorta abdominal (AAA) entre 4 a $5,5 \mathrm{~cm}$ de diámetro mejora la sobrevida con respecto a seguimiento con vigilancia.

\section{Diseño}

Ensayo clínico controlado aleatorizado, multicéntrico.

\section{Lugar}

Centros Médicos de atención de veteranos, EE.UU.

\section{Pacientes}

Los pacientes se identificaron a través de un programa de rastreo por ecografía. Se aleatorizaron 1.136 pacientes de entre 50 a 79 años con AAA asintomáticos.

\section{Intervención}

Cirugía electiva $(n=569)$ vs. vigilancia $(n=567)$. En el grupo vigilancia los pacientes eran seguidos cada seis meses con ecografía o tomografía computada;se indicaba cirugía si comenzaban con síntomas atribuibles al AAA, si el diámetro superaba los $5,5 \mathrm{~cm}$ o si el mismo aumentaba más de $0,7 \mathrm{~cm}$ en seis meses $01 \mathrm{~cm}$ en un año.

\section{Medición de resultados principales}

El resultado principal era mortalidad total medida a partir de los 30 días posteriores a la cirugía. El análisis se realizó por intención de tratar*. Resultados secundarios fueron:tasa de ruptura aneurismática y mortalidad quirúrgica.

\section{Resultados principales}

La media de seguimiento fue 4,9 años.En el grupo cirugía $92,6 \%$ se realizó la operación, en el grupo vigilancia el 61,5 la realizó al final del seguimiento. La tasa de sobrevida en ambos grupos fue similar. Durante el seguimiento ocurrieron 11 rupturas de AAA en el grupo vigilancia (tasa de ruptura anual del 0,6\%). La tasa de crecimiento promedio del AAA fue de $0,3 \mathrm{~cm}$ por año.

\begin{tabular}{l|c|c|c|c} 
Resultados & $\begin{array}{c}\text { Cirugía temprana } \\
(\mathbf{n}=569)\end{array}$ & $\begin{array}{c}\text { Vigilancia } \\
(\mathbf{n}=567)\end{array}$ & $\begin{array}{c}\mathbf{R R} \\
(\mathbf{I C ~ 9 5 \% )}\end{array}$ & $\mathbf{p}$ \\
\hline Mortalidad (n,\%) & $143(25,1)$ & $122(21,5)$ & $1,21(0,95-1,54)$ & NS \\
\hline Ruptura del AAA (n,\%) & $2(0,4)$ & $11(1,9)$ & & \\
\hline $\begin{array}{l}\text { Mortalidad quirúrgica } \\
\text { a30 días ó durante }\end{array}$ & $14(2,7)$ & $7(2,1)$ & & \\
la internación (n,\%) & & & & \\
\hline
\end{tabular}

\section{Conclusión}

A pesar de una muy baja mortalidad quirúrgica, no hay diferencias en la sobrevida comparando cirugía temprana vs. vigilancia con imágenes en pacientes con aneurismas de aorta abdominal menores de $5,5 \mathrm{~cm}$

\section{Comentario}

Los aneurismas de aorta abdominal (AAA) están relacionados con dos procesos: uno de ellos crónico degenerativo causado por alteraciones de la íntima de la pared arterial; y el otro relacionado a la ateroesclerosis. Su combinación causa una debilidad en la pared arterial que predispone a su expansión y ruptura. Los AAA representan la décima causa de muerte en ancianos, y su prevalencia probablemente esté subestimada ya que en un porcentaje alto de los casos debuta como muerte súbita ${ }^{1,2}$.La historia natural de los AAA es una evolución hacia la ruptura, siendo el principal predictor de la misma el diámetro arterial. Es un dato reconocido que aquellos AAA mayores de $5,5 \mathrm{~cm}$ tienen un riesgo de ruptura elevado, por lo que tienen indicación precisa de cirugía electiva. Hasta la publicación de estos ensayos, no era tan claro qué hacer con aquellos de entre 4 y $5,5 \mathrm{~cm}^{3,4}$. En este sentido el resultado de estos estudios resulta muy aclaratorio. Si la ruptura acontece el riesgo de muerte es del $90 \%$, dato que surge del hecho que la mayoría de los pacientes no logran llegar al hospital, y si logran ingresar al quirófano la mortalidad de la cirugía asciende al $50 \%$. El objetivo de la cirugía electiva en pacientes asintomáticos es el de aumentar la expectativa de vida previniendo la ruptura del AAA.Esta elección puede no resultar adecuada, ya que va a depender del riesgo de que la ruptura realmente ocurra, así como de la mortalidad quirúrgica a la que nos enfrentemos. Entonces existe para decidir el tratamiento una ecuación clara: por un lado el riesgo de la cirugía y por el otro e riesgo de ruptura espontánea.Para decirlo en otros términos, las opciones son resolver el problema ahora y como dicen los ciru- janos "desactivar la bomba" (con el consiguiente riesgo de muerte quirúrgica, muy dependiente del centro y país donde trabajemos) o esperar la evolución natural del aneurisma con un criterio de vigilancia activa mediante seguimiento clínico/ecográfico para evaluar expansión y síntomas clínicos de ruptura.

Los dos estudios aleatorizados y controlados analizados comparan la cirugía temprana vs. la vigilancia, o mejor dicho "estrategia de vigilancia clínica y ecográfica". El mayor tiempo de seguimiento del estudio británico (UKSAT), cuyo primer reporte ya fue comentado en Evidencia, no muestra diferencias entre la cirugía electiva y vigilancia después de 8 años de seguimiento. Quedaban dudas en cuanto a qué podía ocurrir si la mortalidad quirúrgica fuese menor al $5,8 \%$ (la encontrada en este estudio).El estudio estadounidense (ADAM) contesta esta pregunta, ya que incluso con una mortalidad oper atoria sensiblemente menor $(2,1 \%)$ la cirugía no mostró ventajas a los 4,9 años de seguimiento medio.

Una de las más importantes lecciones que se pueden aprender de estos estudios es la historia natural de los AAA.Las complicaciones fueron notablemente menores a las registradas en estudios observacionales. La ruptura fue en promedio del $1 \%$ anual y se debió en su mayoría no a rupturas espontáneas si no a que los AAA se expandieron más de $5,5 \mathrm{~cm}$.

Sin embargo, las respuestas no son tan sencillas como parecen. Por un lado encontramos el argumento de que, si tal como sucede en el ADAM, al final del estudio más del $60 \%$ de los pacientes del grupo vigilancia igual van a ser interv enidos.Con toda la ansiedad, costos e inconveniencias que genera ser seguido por una patología que el paciente sabe puede complicarse, sabiendo que al final no hay diferencias. ¿No es mejor operarse de entrada? Además, en 


\section{Aneurismas de aorta abdominal pequeños: no hubo diferencias a largo plazo entre la cirugía y la conducta expectante}

Long term outcomes of inmediate repair compared with surveillance of small abdominal aortic aneurysms. The United Kingdom Small Aneurysm Trial Participants (UKSAT). N Engl J Med 2002;346:1445-52

\section{Objetivo}

Evaluar si la cirugía electiva temprana de pacientes con Aneurismas de Aorta Abdominal (AAA) de tamaño pequeño es superior al seguimiento y vigilancia con ecografía.

\section{Diseño}

Ensayo clínico aleatorizado, controlado, multicéntrico, abier to*.

\section{Lugar}

Realizado en 93 Hospitales del Reino Unido de Gran Bretaña.

\section{Pacientes}

Participaron 1.090 pacientes de entre 60 a 76 años con AAA de entre 4 y $5,5 \mathrm{~cm}$ de diámetro.

\section{Intervención}

Los pacientes fueron aleatorizados a realizar cirugía $(n=563)$ ó vigilancia $(n=527)$. Esta última rama consistía en consultas cada 6 meses con ecografía. Se les ofrecía cirugía si en el seguimiento los AAA excedían los $5,5 \mathrm{~cm}$, si se expandían más de $1 \mathrm{~cm}$ por año o si los AAA se hacían sintomáticos.

\section{Medición de los Resultados Principales}

Mortalidad total, Mortalidad relacionada con AAA, mortalidad quirúrgica. Los médicos que evaluaban las causas de muerte estaban ciegos a la asignación inicial.

\section{Resultados Principales}

Finalizado con una media de seguimiento de 8 años, fueron operados el $92 \%$ de los pacientes en el grupo cirugía y el $62 \%$ en el grupo vigilancia. En el grupo vigilancia, 289 (55\%) se operaron de acuerdo al protocolo y $39(7 \%)$ sin cumplir los criterios del protocolo.

Lo sobrevida media fue de 6,5 años para el grupo vigilancia y 6,7 años para el grupo cirugía temprana $(p=N S)$. El riesgo relativo de muerte total del grupo cirugía comparado con el de vigilancia fue 0,83 (IC 95\%, 0,69 a 1).Las curvas de sobrevida mostraban una clara ventaja inicial para el grupo vigilancia dada una mortalidad quirúrgica del 5,5\%;las curvas se cruzaban a los tres años y a los ocho años la mortalidad era menor para los asignados a cirugía. La mortalidad atribuible a AAA fue $9 \%$ mayor en las mujeres que en los hombres independientemente del grupo inicial asignado. La tasa de cesación tabáquica fue mayor en el grupo cirugía que en el de vigilancia.

\begin{tabular}{|c|c|c|c|c|}
\hline Resultados & $\begin{array}{l}\text { Cirugía temprana } \\
\qquad(\mathrm{n}=563)\end{array}$ & $\begin{array}{c}\text { Vigilancia } \\
(n=527)\end{array}$ & $\begin{array}{c}\text { RR } \\
\text { (IC 95\%) }\end{array}$ & $p$ \\
\hline Mortalidad (n,\%) & $242(43 \%)$ & $254(48 \%)$ & $0,83(0,69-1,00)$ & NS \\
\hline $\begin{array}{l}\text { Muerte por ruptura del } \\
\text { AAA }(n, \%)\end{array}$ & $10(4 \%)$ & $21(8 \%)$ & 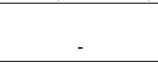 & $<0,05$ \\
\hline $\begin{array}{l}\text { Cirugía de AAA por ruptura } \\
\text { o no ruptura }(n, \%)\end{array}$ & $527(92 \%)$ & $349(62 \%)$ & - & - \\
\hline $\begin{array}{l}\text { Mortalidad quirúrgica a } \\
30 \text { días ó durante } \\
\text { la internación (n,\%) }\end{array}$ & $29(5.5 \%)$ & 28 (7.2\%) & & NS \\
\hline
\end{tabular}

\section{Conclusión}

En pacientes con aneurismas de aorta abdominal pequeños, no se encontraron diferencias a largo plazo entre cirugía inicial o seguimiento con ecografía, si bien a los 8 años de seguimiento la mortalidad fue menor en el grupo de cirugía temprana. Esta diferencia puede ser atribuible en parte a los cambios en el estilo de vida adoptados por este grupo. el "mundo real" ¿puede realizarse o reproducirse una vigilancia tan estrecha o esto se logra solo en ámbitos experimentales especiales como los de un ensayo clínico ${ }^{5}$ ?

Por otro lado puede argumentarse que también en el "mundo real" la mortalidad operatoria es de alrededor del $8 \%$ no del 5,8\%; y mucho menos del $2 \%$. El seguimiento requiere más que nada de un seguimiento clínico así como la evaluación del tamaño del AAA cada seis meses, por lo que es adecuado, factible y de bajo costo. Mi opinión es que la opción según los datos actuales sería tomar AHORA una chance de muerte de $1 / 12$ contra la posibilidad de muerte de 1/100 por año, si en el seguimiento el AAA supera los $5.5 \mathrm{~cm}$ o se complica es de esperar que con el paso del tiempo tanto las técnicas quirúrgicas como el control pre y post operatorio, que son los que inciden en la mortalidad, mejoren.Por lo tanto considero que dado los resultados no me parece muy sensato tomar ahora un riesgo tan alto.

Un aspecto a destacar es la baja cantidad de mujeres en los estudios, ya que si bien los AAA son menos prevalentes en ellas el riesgo de ruptura parece ser mayor.Este sigue siendo un costado gris, probablemente el umbral quirúrgico en mujeres deba ser menor porque anatómicamente poseen aortas más delgadas. Otro aspecto interesante es que en el reporte final del UKSAT al grupo asignado a cirugía le fue ligeramente mejor.Esto no parece atribuirse a la cirugía; la única tendencia claramente diferente fue que el grupo quirúrgico abandonó más el tabaquismo. Nunca debemos olvidarnos que los AAA son en definitiva una enfermedad ateroesclerótica y que como cualquier otra de su tipo, su evolución y probablemente su prevención también está ligada al control de los factores de riesgo ${ }^{7}$.

Conclusiones del comentador: Parece claro que la mejor estrategia con AAA de $4-5,5 \mathrm{~cm}$ es la vigilancia ecográfica semestral; la cirugía electiva no confiere ventajas en cuanto al pronóstico a largo plazo.

\section{Dr. Augusto Granel [ Unidad de Medicina Familiar y Preventiva. Hospital Italiano de Buenos Aires ]}

\section{Referencias}

1-LederleF, Johnson G, Wilson S, et al. Prevalence and associations of abdominal aortic aneurysms detected through screening.Ann Intern Med 1997;126:441-449

2-Gillum RF.Epidemiology of aortic aneurysm in the United States.J Clin Epidemiol 1995:48:1289-98

3-Manfredini R, Portaluppi F, Grandi E, et al.Out of Hospital sudden death referring to an emrgency department. J Clin Epidemiol 1996;49:865-68

4-Nevitt MP, Ballard DJ, Havitt JW.Prognosis of abdominal aortic aneurysms: a population based study.N Engl J Med 1989;231:1009-14

5-Valentine RJ, De Caprio JD, Castillo Jm , et al.Watchfull waiting in cases of small abdominal aortic aneurysms, appropiate for all patientes? J Vasc Surg 2000;32:441-450

6-Zarins CK, Harris EJ. Operative repair for abdomianl aortic aneurysms: the gold standard.J Endovasc Surg 1997,4:232-241

7- Brady AR, Fopwkes FGR, Greenhald , et al..Risk factors or postoperative death following elective surgery repair of abdominal aortic aneurysm: results from de UKSAT.Br J Surg 2000;87:742-49 